\title{
Clinical Profile of TB Patients on DOTS at a Tertiary Care Hospital
}

\author{
Arjun $\mathrm{H}^{1}$, Santosh VG \\ ${ }^{1}$ Assistant Professor, Department of Pulmonary Medicine, JJMMC, Davangere, Karnataka.
}

\section{Abstract}

Background: Tuberculosis was considered to be among the top ten causes of global mortality and morbidity. The highest incidence of TB was found in developing countries. Estimates suggest that $32 \%$ of the worlde's population was infected with TB. In India, it was seen that TB kills more adults than any other infectious disease. $20 \%$ global burden of TB was in India. Subjects and Methods: Before initiating antituberculosis treatment a detailed clinical assessment which included history and clinical examination was done. Hematological investigations like HIV test is done as per ICTC along with HBsAg. Hematological investigations included hemoglobin, WBC count, differential count, platelet count, prothrombin time and ESR. Biochemical investigations included total bilirubin, direct bilirubin, AST, ALT, alkaline phosphatase, random blood sugar, blood urea, s.creatinine and uric acid. Results: Out of the 50 patients studied, $70 \%$ patients were sputum positive and $30 \%$ were sputum negative. This is probably because majority of the patients studied were pulmonary tuberculosis. Conclusion: Adverse drug reaction was seen in 29 patients $(58 \%)$. One or more types of ADR was seen in these patients.

Keywords: TB patients, DOTS, HIV.

Corresponding Author: Dr. Arjun H, Assistant Professor, Department of Pulmonary Medicine, JJMMC, Davangere, Karnataka.

Received: July 2019

Accepted: July 2019

\section{Introduction}

The World Health Organization declared tuberculosis (TB) as a global emergency in 1993. The number of TB cases has increased since 1983 due to HIV/AIDS. India in considered to be in a unique position with respect to the global tuberculosis epidemic. A comprehensive review of the tuberculosis program in India in 1992 found that less than half of the patients with tuberculosis received an accurate diagnosis and that less than half of those were effectively treated. Tuberculosis was considered one of the foremost public health problems causing an enormous burden of suffering and deaths. Chemotherapy was considered the basic approach to clinical tuberculosis control. Essential antituberculosis drugs are Isoniazid (INH), Rifampicin (RMP), Pyrazinamide (PZA), Ethambutol (E) and Streptomycin (S). Directly observed treatment, short-course (DOTS) was the brand name of the WHO recommended TB control strategy. It was vital for the success of TB control, that the, health care workers should treat TB patients within the framework of Revised National Tuberculosis Control Programme (RNTCP)..$^{[1]}$

Tuberculosis was considered to be among the top ten causes of global mortality and morbidity. The highest incidence of TB was found in developing countries. Estimates suggest that $32 \%$ of the world's population was infected with TB. In India, it was seen that TB kills more adults than any other infectious disease. $20 \%$ global burden of TB was in India. ${ }^{[2]}$ South East Asia dominates the worldwide distribution of notified cases (36\% of the total cases). The global rate of tuberculosis was growing at approximately $1.1 \%$ per year. India ranks first in the estimated number of tuberculosis cases, and approximates to 1761 (thousands) cases per 10, 49,549 population at the rate of 168 cases per $1,00,000$ population. ${ }^{[3]}$

India was considered the highest $\mathrm{TB}$ burden country accounting for one fifth of the global incidence (Global annual incidence estimate was 9.1 million cases out of which it was estimated that 1.9 million cases were from India). India was 17th among 22 High Burden Countries in terms of TB incidence rate.

Diagnosis was primarily by sputum microscopy, treatment was directly observed, and standardized regimens and methods of recording and reporting were used. It was gradually expanded to cover a population of 20 million by mid-1998. Rapid RNTCP expansion began in late 1998. By the end of $2002,50 \%$ of country"s population was covered under RNTCP. ${ }^{[4]}$

The country was fully covered by 23rd march 2006. Every day in India, under the RNTCP more than 5000 patients were examined for TB, free of charge. As a result of these examinations every day, more than 3200 patients were started on treatment and more than 2500 of them were cured. RNTCP was considered the largest and the fastest expanding programme in the world. ${ }^{[5,6]}$

\section{Subjects and Methods}

50 tuberculosis patients who were started on DOTS were selected for the study using purposive sampling technique. 


\section{Inclusion Criteria:}

- Patients with newly diagnosed pulmonary or extrapulmonary tuberculosis who were started on DOTS.

- Age $>15$ years

\section{Exclusion Criteria:}

- Patients who had clinical jaundice before initiating treatment.

- Patients who had elevated levels of bilirubin, AST, ALT, ALP, blood urea, serum creatinine, uric acid before initiating treatment.

- Patients with thrombocytopenia.

\section{Method of collection of data:}

Before initiating anti-tuberculosis treatment a detailed clinical assessment which included history and clinical examination was done. Hematological investigations like HIV test is done as per ICTC along with $\mathrm{HBsAg}$.

Hematological investigations included hemoglobin, WBC count, differential count, platelet count, prothrombin time and ESR. Biochemical investigations included total bilirubin, direct bilirubin, AST, ALT, alkaline phosphatase, random blood sugar, blood urea, s.creatinine and uric acid.

Clinical evaluation, hematological tests as well as biochemical tests were done pre-treatment, 1st week, 1st month and 4th month.

Patients were instructed to report any symptoms they would experience during the course of treatment. Symptoms looked for during the follow up period were fever, nausea, vomiting, anorexia, bitter taste sensation, loss of taste, abdominal pain, diarrhoea, lethargy, myalgia, arthralgia, jaundice, bleeding diathesis, photosensitivity, itching, rashes, confusion, blurring of vision, tingling and numbness, dizziness, loss of balance, convulsions, psychosis and depression. Physical examination included skin rashes, bleeding manifestations, jaundice, hepatomegaly, hepatic tenderness, ascites, edema, signs of peripheral neuropathy.

\section{Data - Analysis}

Statistical analysis was done by analysis of variance (ANNOVA) to analyse pretreatment versus post treatment values at 1 st week, 1 st month and 4 th month.

\section{Results}

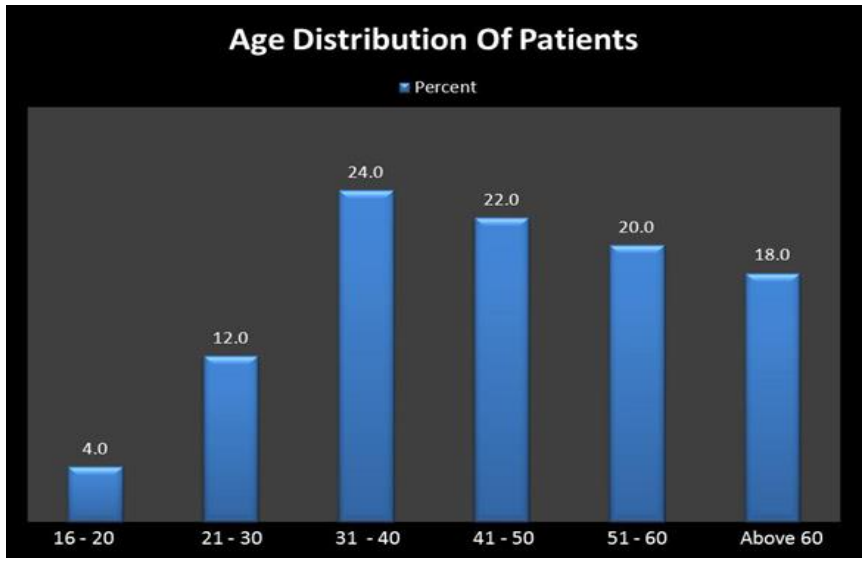

Figure 1: Age distribution of patient
Out of the 50 patients studied majority of patients were in the age group of 31-40 years which constituted $24 \%$, followed by $22 \%$ among $41-50$ and $20 \%$ 51-60 yrs.

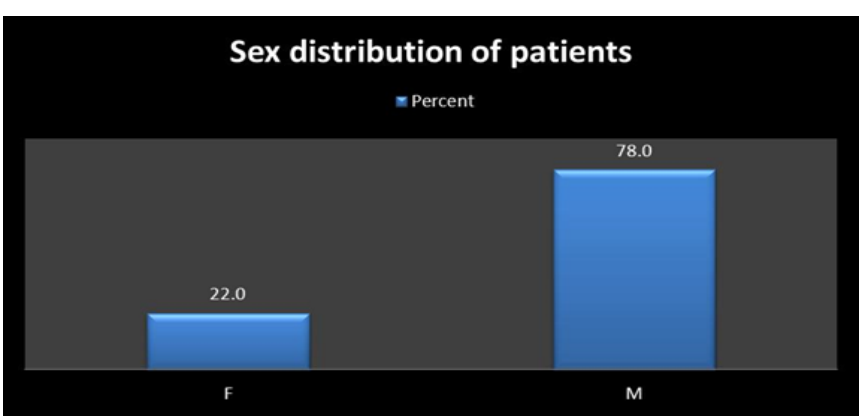

Figure 2: Sex distribution of patients

In this study population, out of the 50 patients studied 39 (78\%) were males and $11(22 \%)$ were females. The male to female ratio was 3.5:1.

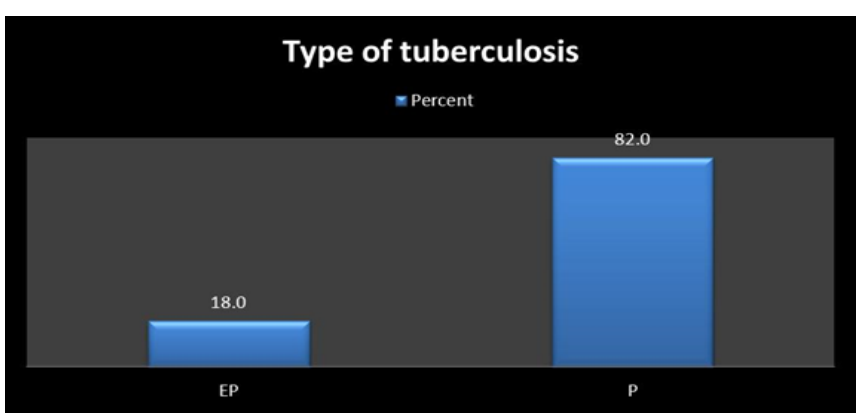

Figure 3: Type of tuberculosis

Pulmonary tuberculosis constituted $82 \%$ of the study group and $18 \%$ were extrapulmonary tuberculosis.

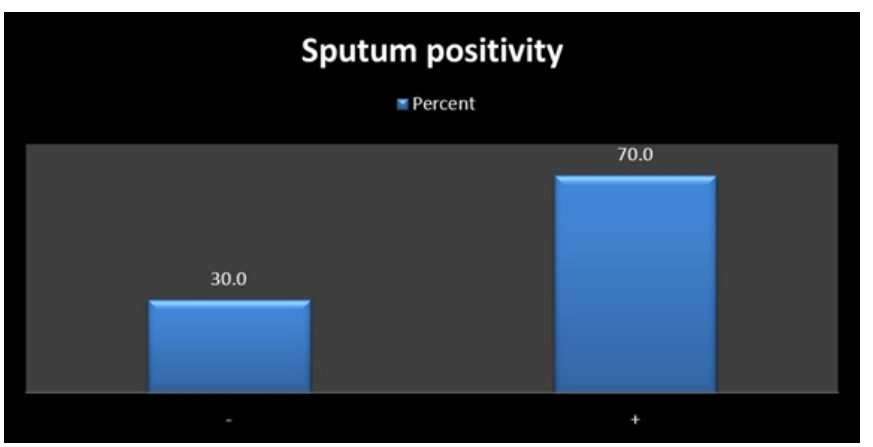

Figure 4: Sputum positivity

Out of the 50 patients studied, $70 \%$ patients were sputum positive and $30 \%$ were sputum negative. This is probably because majority of the patients studied were pulmonary tuberculosis.

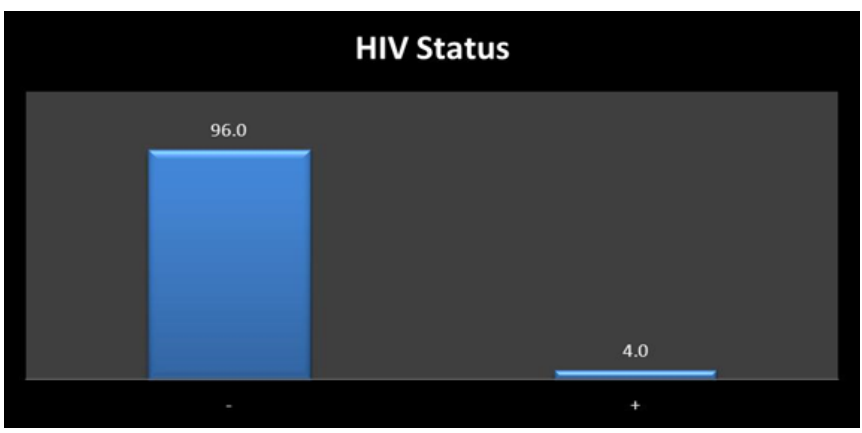

Figure 5: HIV status 
In this study group, 2 patients (4\%) were HIV positive.

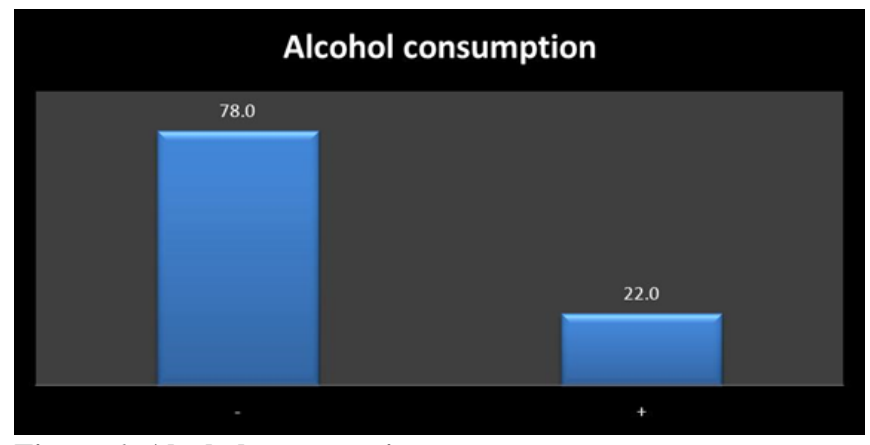

Figure 6: Alcohol consumption

Out of the 50 patients, 11 patients (22\%) consumed alcohol.

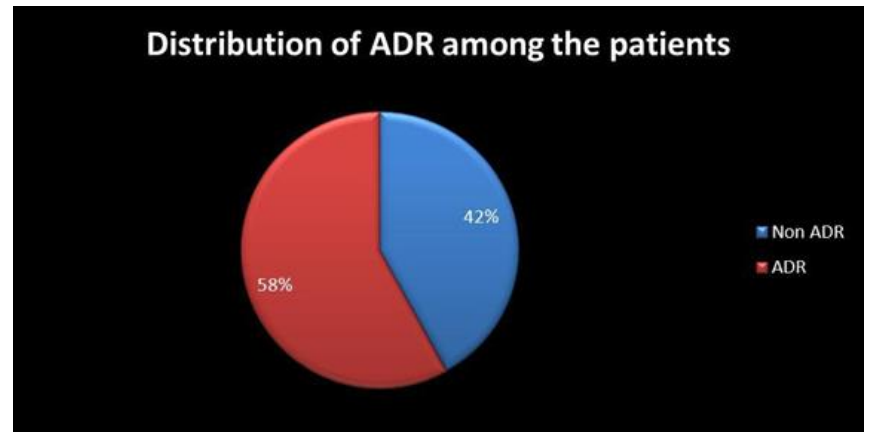

Figure 7: Distribution of ADR among the patients

Adverse drug reaction was seen in 29 patients (58\%). One or more types of ADR was seen in these patients.

\section{Discussion}

Before the advent of the DOTS programme, high prevalence countries like India had a National TB Programme to combat the problem of TB. India's National Tuberculosis Programme (NTP) was started in 1962 (NTP employs a daily regimen of anti-TB drugs) was a truly integrated programme, implemented through District Tuberculosis Centres (DTCs) and peripheral health institutions. After more than three decades, the NTP was made notable, but not spectacular progress. The overall case finding programmes were about $33 \%$ and treatment efficiency is of the same order or In order to intensify the efforts to control TB, the Government of India gradually replaced NTP by the DOTS strategy/programme in 1993 and it was known as the Revised National Tuberculosis Programme (RNTCP). The objective of this revised strategy was to achieve a cure rate of $85 \%$ for infections and seriously ill patients through intermittent (three days a week) supervised short course chemotherapy or the directly observed treatment, short course (DOTS). 7 Under RNTCP, the doses of first line anti-TB drugs (Isoniazid, Rifampicin, Pyrazinamide, Streptomycin and Ethambutol) were standardized on the basis of body weight and were given in different regimens. All regimens had an initial intensive phase lasting 2-3 months, aimed to rapidly kill the TB bacilli, bring about sputum conversion and to afford symptomatic relief. This was followed by a continuation phase lasting 4-6 months, during which the remaining bacilli were eliminated so that relapse does not occur. In spite of implementation of DOTS, it is still a major health concern. ${ }^{[8]}$

Adverse drug reactions to anti tuberculosis drugs occured during treatment of tuberculosis and was expected in $10 \%$ of the patients treated for pulmonary tuberculosis. Adverse drug reactions were however more common and often severe in patients on second line drugs. In patients who received conventional therapy of long duration, many patients defaulted when their symptoms ameliorated, but some of them left treatment and became non-adherent due to adverse toxic reactions of drugs. The DOTS therapy based on powerful bactericidal drugs shortened the treatment duration and increased compliance and adherence with an additional advantage of lowering drug toxicity mainly because of fewer doses and less cumulative doses of drugs. Serious side effects of the anti-tuberculosis drugs were known to be less with intermittent therapy. A study by Dhingra and others reported that approximately $8.4 \%$ of the patients treated under DOTS complained of some sort of adverse drug reaction and that most of them occurred during first four weeks in the intensive phase of treatment. Most of the drug reactions were not serious and were managed by symptomatic treatment. It was usually not advisable or even required to stop anti tuberculosis drugs since most of the drug side effects were minor and could be managed by symptomatic treatment or by modifying the administration schedule. In 2-3 weeks the patients usually get adapted and adjusted to the treatment regimen. ${ }^{[9]}$

A major side effect was defined as any adverse reaction that resulted in discontinuation of one or more drugs, and/or directly resulted in hospitalization. A major adverse reaction to one of the first-line antituberculosis drugs, which results in discontinuation of that drug, had several implications. ${ }^{[10]}$

Antitubercular drugs, just like other drugs used in clinical practice, were not free from ADRs. The added problem is that combinations of drugs were always used for prolonged periods of time and therefore, it was likely that the adverse reactions of one drug may be potentiated by the companion drugs used. Moreover, the Adverse Drug Reactions (ADRs) to the drugs used was one of the major reasons for the patient default for treatment. ${ }^{[11]}$

Major adverse reactions to antituberculosis drugs caused significant morbidity, and compromised treatment regimens for tuberculosis (TB). Side effects, resulting in modification or discontinuation of therapy, or hospitalization, were attributed on the basis of resolution after withdrawal, and/or recurrence with rechallenge.

It was seen that there may be considerable morbidity, even mortality, particularly with drug-induced hepatitis. These events were seen to incur substantial additional costs because of added outpatient visits, tests, and in more serious instances hospitalizations. Alternative agents were seen to have greater problems with toxicity, and were often less effective, so that treatment must be prolonged, with attendant challenges to ensure compliance. As a result the risk of treatment failure and relapse were higher. A general knowledge of the various ADRs and their management was considered essential for the effective management of TB. All antitubercular drugs were known to cause adverse drug reactions6 and may result in ADRs involving almost all systems in the body, including 
the gastrointestinal tract, liver, skin, nervous system, otovestibular apparatus and the eyes. ${ }^{[12]}$

\section{Conclusion}

- A total of 50 patients were selected for this study out of which $78 \%$ were males and $22 \%$ were females, and maximum distribution (of $24 \%$ ) was seen in the age group of 31-40 yrs.

- Pulmonary tuberculosis constituted $82 \%$ and extrapulmonary tuberculosis was $18 \%$ of the study group.

- Out of the 50 patients, 29 patients developed atleast one or more types of ADR.

\section{References}

1. Enarson DA, Rieder HL, Arnadottir T, Trebucq A. Tuberculosis Guide for Low Income Countries. 4th ed. International Union against Tuberculosis and Lung Disease 1996.

2. Murray CJ, Lopez AD. Mortality by Cause for Eight Regions of the World: Global Burden of Disease Study. Lancet 1997; 349 (9061): 1269-76

3. Murray CJ, Lopez AD. Regional Patterns of Disability Free Life
Expectancy and Disability - Adjusted Life Expectancy: Global Burden of Disease Study. Lancet 1997; 349 (9062): 1347 -52

4. World health organization. Global Tuberculosis control: Surveillance, Planning, Financing. WHO report 2005. Geneva: World Health Organisation, 2005

5. RNTCP status report DOTS for ALL, ALL for DOTS TB INDIA 2006, Central TB Division, Directorate General of Health Services MHFW New Delhi.

6. World Health Organization, International Union against Tuberculosis and Lung Disease, Royal Netherlands Tuberculosis Association. Revised international definitions in tuberculosis control. Int $\mathbf{J}$ Tuberc Lung Dis 2001; 5:213-5.

7. Cantwell MF, Binkin NJ. Tuberculosis in Subsaharan Africa: A Regional Assessment of the Impact of the Human Immuno Deficiency Virus and National Tuberculosis Control Programme Quality. Tuber Lung Dis 1996; 77 (3): 220-5

8. RNTCP status report I am stopping TB, TB INDIA 2009 Central TB Division, Directorate General of Health Services MHFW New Delhi.

9. Treatment of Tuberculosis: Guidelines for National Programmes. 2nd ed. Geneva: World Health Organization, 1997.

10. Shashikant. Control of Tuberculosis. In: Sharma SK, Mohan, Tuberculosis. New Delhi: Jaypee Brothers 2004; 556-8.

11. Tripathi KD. Antitubercular drugs, Essentials of Medical Pharmacology. 5th ed. New delhi: Jaypee Brothers, 2003: 705-8.

12. Sharma H, Aqil M, Imam F, Alam MS, Kapur P, Pillai KK. A Pharmacovigilance Study in the Department Of Medicine of a University Teaching Hospital. Pharmacy Practice 2007; 5(1): 46-9.

Copyright: (C) the author(s), 2019. It is an open-access article distributed under the terms of the Creative Commons Attribution License (CC BY 4.0), which permits authors to retain ownership of the copyright for their content, and allow anyone to download, reuse, reprint, modify, distribute and/or copy the content as long as the original authors and source are cited.

How to cite this article: Arjun H, Santosh VG. Clinical Profile of TB Patients on DOTS at a Tertiary Care Hospital. Acad. J Med. 2019;2(2):1-4.

DOI: dx.doi.org/10.21276/ajm.2019.2.2.1

Source of Support: Nil, Conflict of Interest: None declared. 